\title{
Treatment of Intractable Non-Rapid Cycling Bipolar Affective Disorder with High-Dose Thyroxine: An Open Clinical Trial
}

Andreas Baumgartner, M.D., Michael Bauer, M.D., Ph.D., and Rainer Hellweg, M.D.

Six patients with very severe forms of non-rapid cycling bipolar affective illness whose symptoms had previously been refractory to all current antidepressant and/or prophylactic medications were treated with supraphysiological doses of thyroxine ( 250 to $500 \mu \mathrm{g} /$ day) as an adjuvant to their previous medications. The mean follow-up period was $27.8 \pm 12.8$ months (range 12 to 46). The mean number of relapses during the follow-up period of each patient declined from $5.3 \pm 3.1$ to $0.8 \pm$ 0.8 and the mean duration of hospitalization from $10.0 \pm 5.6$ to $0.8 \pm 1.2$ months as compared to the same length of time for each patient before the start of treatment with high-dose thyroxine (T4). Three of the patients had no further relapses at all. Thus, for these patients, who had previously been severely ill and therapy-resistant, high-dose T4 administration proved to have excellent effects on the course of the illness. However, in five of these patients the effect of the T4 was strong enough only when it was administered in combination with a prophylactic and antidepressant and/or neuroleptic drug, of which in some cases high doses were also needed. The side effects were negligible. Mechanisms that may possibly underlie the beneficial effects of high-dose T4 in bipolar affective disorder are discussed. [Neuropsychopharmacology 10:183-189, 1994]
KEY WORDS: Bipolar affective disorder; Treatment resistance; Thyroxine treatment

Numerous interactions have been reported between thyroid function and affective disorders. Severe forms of thyroid disorder such as hypo- or hyperthyroidism may induce all kinds of manic and depressive symptoms (for a review, see Hall 1986). On the basis of these clinical observations, as early as the 1960s Prange and coworkers began systematically investigating whether treatment with thyrotropine-releasing hormone (TRH), thyrotropine (TSH) or directly with triiodothyronine (T3) had any value in the treatment of depressed pa-

From the Psychiatrische Klinik and Poliklinik, Universitätsklinikum Rudolf-Virchow (Charlottenburg), Free University of Berlin, Berlin, Germany.

Address all correspondence to: Dr. A. Baumgartner, Psychiatrische Klinik, Eschenallee 3, D-14050 Berlin, Germany.

Received August 31, 1993; revised December 20, 1993; accepted January 3, 1994. tients (Prange et al. 1969, 1970, 1972). However, the beneficial effects of TRH and T3 supplementation of current antidepressant medication or in treatment-resistant patients remain controversial to date. In regard to the efficacy of T3, in particular, both beneficial (e.g., Goodwin et al. 1982; Joffe and Singer 1990) and negative results (e.g., Feighner et al. 1972, Gitlin et al. 1987, see Stein and Avne 1988 for a review) have been reported.

During the past decade three reports have been published that describe beneficial effects of treatment with high, supraphysiological doses of thyroxine (T4) in previously treatment-resistant, rapid-cycling, bipolar affective illness. Stancer and Persad (1982) reported that rapid cycling ceased in five out of eight women, but did not in two men, following treatment with up to $500 \mu \mathrm{g}$ T4 per day. Leibow (1983) reported on the case of a rapid-cycling woman who responded well to $400 \mu \mathrm{g}$ thyroxine per day. Finally, Bauer and Whybrow (1990) published impressive results of treatment with high-dose T4 (150 to $400 \mu \mathrm{g} /$ day) in patients with rapid- 
cycling affective illness who had previously failed to respond to any treatment.

All of these studies intentionally included rapidcycling, bipolar patients only. As rapid cycling affects only approximately 10 to $15 \%$ of all bipolar patients (e.g., Dunner and Fieve 1974), which in turn is only a small minority of patients with affective illness, we were interested in investigating whether administration of high-dose T4 would also be effective in treatmentresistant, non-rapid cycling, bipolar patients. Here we report the initial results of an ongoing open trial of highdose T4 treatment in six previously treatment-resistant, very severely ill bipolar patients. The results for the furst 4 years of the trial (1989-1993) are given.

\section{PATIENTS AND METHODS}

The patients were accepted for the study on the basis of both their diagnosis and their clinical course. They were required to meet DSM-III-R criteria for major affective disorder, bipolar subtype. However, patients fulfilling the criteria established by Dunner and Fieve (1974) for rapid cycling (i.e., four or more affective episodes within the 12 months immediately preceding evaluation) were not accepted. As little experience has been gained to date with long-term treatment of depressed patients with supraphysiological doses of T4, we could not rule out the possibility that side effects might occur and that they might even be severe, particularly during chronic treatment. For ethical reasons we therefore deliberately selected only patients who had been resistant to all conventional medication and whose illness had taken a very severe course during the past few years. Thus, only patients who failed to respond to prophylactic treatment with lithium alone, carbamazepine alone, a combination of both, or a combination of these prophylactic drugs with antidepressants and/or neuroleptics were considered for inclusion in the study. A patient was classified as treatmentresistant only if treatment with each of the above drugs or combinations of drugs had been unsuccessful for at least 6 months. Since 1989 a total of 11 patients have taken part in the study. In two cases high-dose T4 treatment was not instituted until after July 1992; thus it is too early to assess the clinical efficacy of the treatment in these two patients. A further two patients were withdrawn from the study because of non-compliance. They stopped taking both thyroxine and their other psychotropic medication several times during the first year of treatment, as they had also done during the period before beginning high-dose T4 treatment. A fifth patient, who had delusional depression (bipolar II) broke her hip in a fall and was treated for this and other organic disorders in other hospitals, and thus it was not possible to continue with the high-dose T4 treatment. Therefore, a total of six patients finally took part in the study. Their clinical and sociodemographic data are shown in Table 1.

Four of these patients were women and two were men, and their mean age was 52.8 years with a standard deviation of 11.8 and a range of 33 to 68 years. All of them had major affective disorder, which was bipolar I subtype in four cases and bipolar II subtype in two. None of these patients fulfilled the DSM-III-R definition or the Dunner and Fieve criteria (Dunner and Fieve 1974) for rapid cycling (see Table 1). In none of the cases did the reasons for instituting high-dose T4 treatment constitute an attempt to handle a treatmentresistant course of rapid-cycling major affective disorder, as was the case in all previous studies (Stancer and Persad 1982; Leibow 1983; Bauer and Whybrow 1990). The six patients were included in the study for the following reasons. Patients 1,3 , and 4 had repeated episodes of treatment-resistant depression. Their depressive symptoms had repeatedly failed to respond to high doses of different antidepressants, with and without lithium and/or carbamazepine treatment. They sometimes responded to a series of electroconvulsive therapy (ECT), but the depressive symptomatology regularly returned after periods varying from some weeks or, at best, some months. As can be seen from Table 1 , during the 3-year period prior to the start of treatment with $\mathrm{T} 4$ these patients were hospitalized for 3 , 18 , and 16 months, respectively. They were depressed for most of the time, and hypomanic episodes (e.g., after ECT treatment) were the exception. In these three patients the main purpose of administering high doses of $\mathrm{T} 4$ was therefore to prevent their most severe and resistant relapses into depression.

In contrast, patients 2,5 , and 6 also had severe manic episodes that always required hospitalization on a locked ward and had been involved several times in conflicts with the police or other institutions. The symptomatologies of patients 2 and 6 were predominantly manic, with only short depressive episodes of minor severity. All prophylactic agents (lithium, carbamazepine, valproate [in two patients] and neuroleptics) failed to prevent further manic episodes in these patients. Over a period of several years this severe course of the illness had led to severe impairment of the social and working lives of all of these patients. For these reasons we instituted high-dose T4 treatment in an attempt to prevent further severe manic episodes. None of the patients had a history of alcohol or drug abuse or was currently addicted to any substance. However, the four women had histories of subclinical hypothyroidism and/or Hashimoto's thyroiditis (patients 1 through 4, Table 1). In three of these women, the thyroid disorder clearly preceded the initiation of lithium treatment, and 


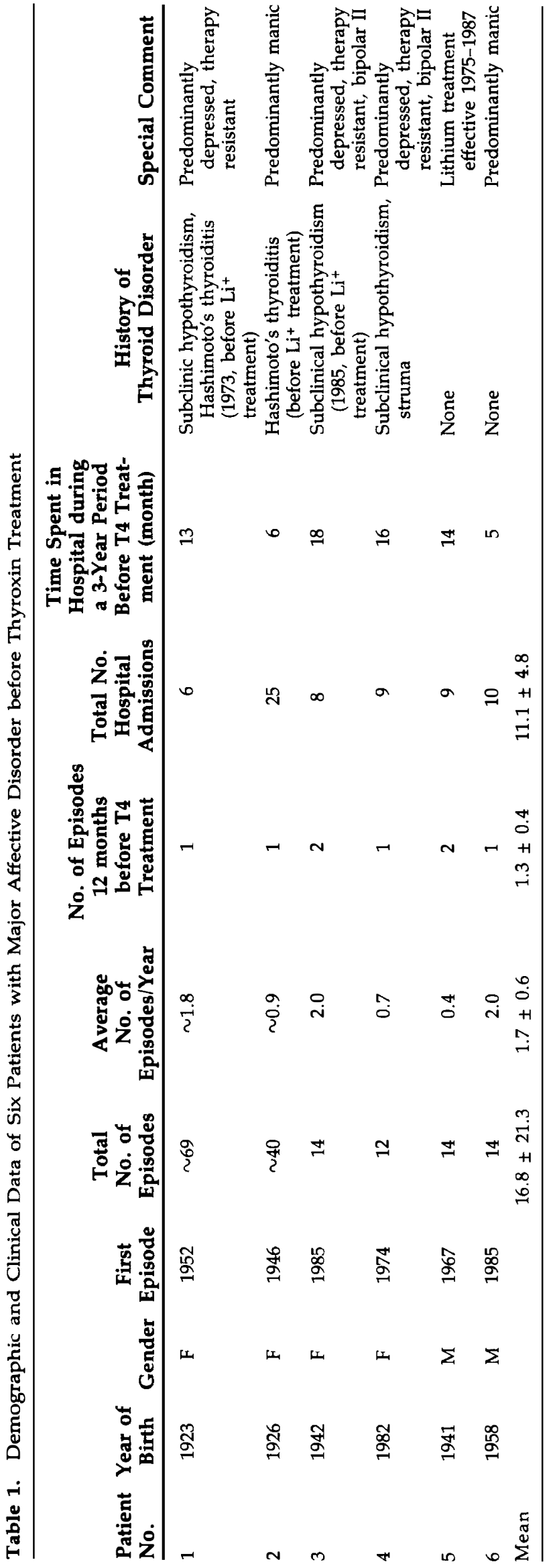

in the fourth it was no longer possible to ascertain whether there was any temporal relationship. Three of them (patients 1, 2, and 3) had received physiological doses of T4 (100 to $150 \mu \mathrm{g} /$ day) in the years preceding the institution of high-dose T4 therapy. The fourth patient had received $100 \mu \mathrm{g}$ T4/day from 1980 to 1985 only.

As the report by Bauer and Whybrow (1990) emphasized, because the administration of $\mathrm{T} 4$ is probably more efficient in combination with conventional prophylactic drugs, we added T4 to the previous medications of all patients. We later made several attempts to reduce or withdraw the concomitant psychotropic drugs (see Results). The T4 was instituted at a starting dose of $50 \mu \mathrm{g}$ and was increased in weekly $50-\mu \mathrm{g}$ increments. A complete "thyroid status" (serum concentrations of thyroxine, fT4, T3, fT3, baseline TSH) and an electrocardiogram were obtained three times a week until the final dose was reached, and sustained for at least four weeks. The dose was increased until the serum patient's T4 levels showed a distinct rise to between 150 and $200 \%$ of the initial levels. Another criterion for deciding whether the dose should be reduced further or raised, was the occurrence of severe subjective or objective side effects, such as tachycardia and severe weight loss. However, it was not necessary to stop increasing the doses for any patient before the desired serum level of T4 was reached (see above) because of side effects (the only patient whose previous dose of $250 \mu \mathrm{g} /$ day had to be reduced, because of severe weight loss and disturbed sleep, was one of those who dropped out, see above).

The efficacy of high-dose T4 treatment was evaluated as follows. An "episode" or a "relapse" was defined as a change in a patient's affective state that required either changes in medication on an outpatient basis (e.g., increases in the doses of the drugs taken), a change to new drugs, or hospitalization. Furthermore, the total time (in months) spent in hospital following institution of high-dose T4 administration was compared to the time spent in hospital before the high-dose prophylaxis was begun, separately for each patient (i.e., when we were able to follow a patient on high-dose T4 for 36 months [e.g., patient 1, Table 2], we compared the duration of hospitalization during these 36 months with that during the 36 months prior to institution of the high-dose T4 treatment).

During a relapse all patients were rated weekly on the Bech-Rafaelsen Scale. When not in hospital, the patients were seen regularly by one of the authors at one of our outpatient clinics. During relapses the patients were seen at least once a week and during euthymic phases approximately once every 4 to 6 weeks. Serum levels of lithium and/or carbamazepine were monitored regularly before and during the high-dose T4 treatment and maintained within the prophylactic range in all 


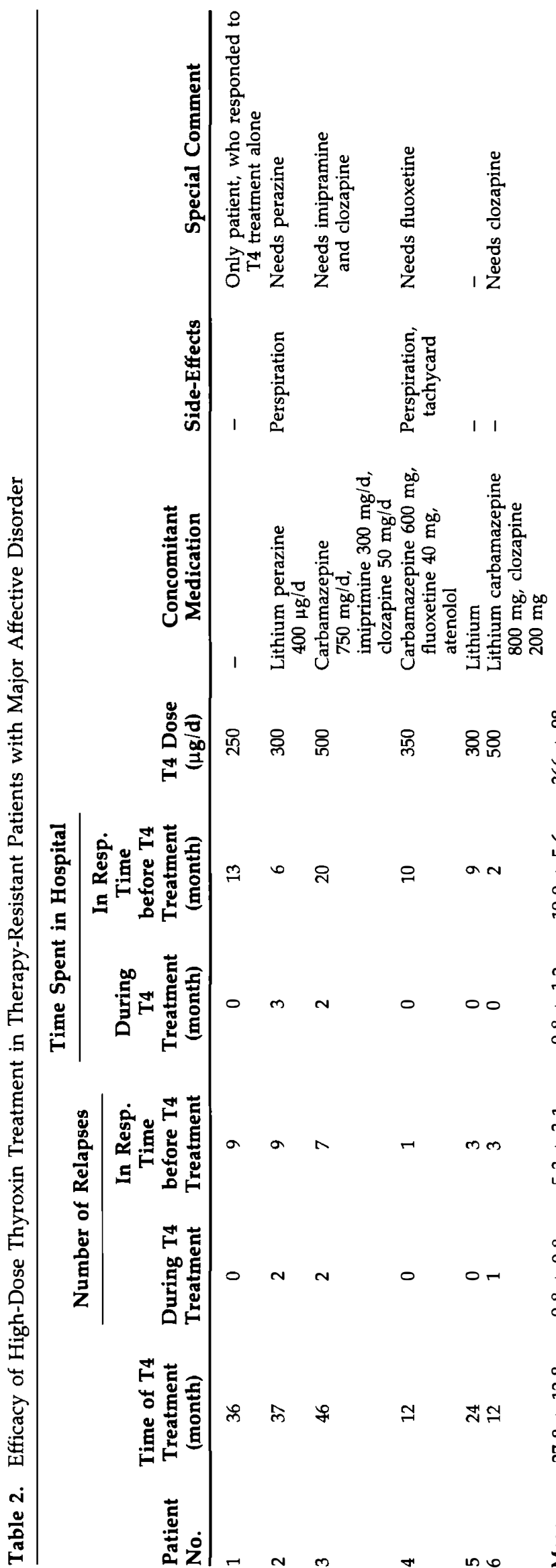

cases. The concentrations of TSH and thyroid hormone (T4, fT4, T3, and fT3) were determined at least once every 6 weeks during euthymic phases and once a week during relapses. The hormone determinations were carried out as previously described with IRMA (for TSH), RIA (T4 and T3) and RIA-Dynotest (for fT3 and fT4 respectively) (Baumgartner et al. 1988). Kits from Henning, Berlin were used throughout. All data are given as means \pm standard deviations.

\section{RESULTS}

The mean follow-up time for all six patients was 27.8 \pm 12.8 months (range 12 to 46 months) (Table 2). During this period the mean number of relapses was 0.8 \pm 0.8 (range 0 to 2 ) as compared to $5.3 \pm 3.1$ (range 1 to 9) in the respective periods prior to institution of high-dose $\mathrm{T} 4$ treatment (Table 2). Four of the patients were able to stay out of hospital completely during the high-dose $\mathrm{T} 4$ treatment and two others were readmitted for a total of 2 and 3 months respectively (see below). Table 2 shows that the mean time spent in hospital was reduced from $10.0 \pm 5.6$ months (range 6 to 20 months) before $\mathrm{T} 4$ treatment to $0.8 \pm 1.2$ months (range 2 to 3 months) for the same length of time during administration of high-dose T4. Thus, the mean number of relapses during $\mathrm{T} 4$ administration was equal to only approximately $15 \%$ of the number occurring during the period prior to institution of $\mathrm{T} 4$ treatment, and the mean duration of hospitalization was approximately $8 \%$ of the duration before the start of the $\mathrm{T} 4$ prophylaxis.

Attempts were made to reduce or even withdraw the concomitant psychotropic medication for all patients except patient 5 . In patient 2 the hypomanic symptoms recurred even when the $400 \mathrm{mg} /$ day dose of perazine was reduced to $200 \mathrm{mg} /$ day. In contrast to the period before high-dose $\mathrm{T} 4$ treatment, these symptoms subsided within days of raising the perazine dose to 400 to $500 \mathrm{mg} /$ day again. Thus, the two relapses of this patient (see Table 2) were obviously related to a reduction of the neuroleptic dose.

The relevance of concomitant psychotropic medication for patient 3 is particularly well documented. This patient had two brief depressive relapses even after she had already been taking $500 \mu \mathrm{g}$ T4/day for several months. At the time of the first relapse she was also taking $100 \mathrm{mg}$ imipramine/day. This dose was increased to $150 \mathrm{mg} /$ day during a stay in hospital. Following discharge from hospital and some months during which she was euthymic, she had a further relapse into depression, which again required hospitalization. This time the dose of imipramine was increased to $300 \mu \mathrm{g} /$ day, and since then she has had no further depressive episodes. Note that it was possible to successfully treat the 
two episodes of depression that occurred during highdose T4 treatment within one month in each case, whereas before high-dose $\mathrm{T} 4$ supplementation the average duration of hospitalization required to treat this patient's depressive episodes was about 5 to 6 months.

We tried twice to withdraw concomitant low-dose (50 to $100 \mathrm{mg} / \mathrm{kg}$ ) clozapine medication in this patient. Both times she reported sleep disturbances beginning on the night immediately after clozapine withdrawal and felt that she was in danger of developing manic symptoms. Therefore, after two days the clozapine was reinstituted on both occasions.

Finally, we realized that patient 4 needed at least $40 \mathrm{mg} /$ day fluoxetine in order to prevent depressive relapses and patient 6 developed hypomanic symptoms when we tried to reduce his clozapine dose from 200 $\mathrm{mg} /$ day.

In summary, only patient 1 has remained on T4 alone $(250 \mu \mathrm{g} /$ day $)$ without further relapses for a total of exactly 3 years. In fact, our subjective impression was that she began to feel really well only after the lithium, carbamazepine and, finally, valproate had been withdrawn.

For all serum levels of thyroid hormones we calculated the mean values of the three serum level determinations conducted before institution of high-dose T4 administration and compared them with the means of the three determinations performed for each patient at the end of the follow-up period. The mean serum T4 levels for the whole group of six patients before highdose $\mathrm{T} 4$ treatment was $81.0 \pm 23.0 \mu \mathrm{g} / \mathrm{L}$ (range: 60 to $120 \mu \mathrm{g} / \mathrm{L}$ ). At the end of the follow-up period, this value had increased to $155.8 \pm 22.4 \mu \mathrm{g} / \mathrm{L}$ (range: 130 to 200 $\mu \mathrm{g} / \mathrm{L}$ ). Thus, the mean serum levels of T4 had approximately doubled. The normal range for serum concentrations of T4, established by our own laboratory from multiple determinations in healthy subjects, was between 48 and $120 \mu \mathrm{g} / \mathrm{L}$.

The mean concentrations of fT4 rose from $10.6 \pm$ $2.8 \mathrm{ng} / \mathrm{L}$ (range: 7.7 to $15 \mathrm{ng} / \mathrm{L}$, normal range: 8 to 19 $\mathrm{ng} / \mathrm{L}$ ) before the start of high-dose T4 treatment to $22.6 \pm$ $5.3 \mathrm{ng} / \mathrm{L}$ (range: 21 to $30 \mathrm{ng} / \mathrm{L}$ ). The serum levels of T3 were $1.01 \pm 0.25 \mu \mathrm{g} / \mathrm{L}$ (range: 0.7 to $1.5 \mu \mathrm{g} / \mathrm{L}$, normal range: 0.7 to $1.8 \mu \mathrm{g} / \mathrm{L}$ ). At the end of the follow-up periods, the mean serum concentration of T3 for the whole group of patients had increased to $1.63 \pm 0.44$ $\mu \mathrm{g} / \mathrm{L}$ (range: 1.1 to $2.5 \mu \mathrm{g} / \mathrm{L}$ ). The levels of fT3 were $3.3 \pm 9.4 \mathrm{ng} / \mathrm{L}$ (range: 1.8 to $4.3 \mathrm{ng} / \mathrm{L}$, normal range: 2.2 to $5.5 \mathrm{ng} / \mathrm{L}$ ) before high-dose T4 treatment and $4.9 \pm$ $9.6 \mathrm{ng} / \mathrm{L}$ (range: 3.6 to $6.4 \mathrm{ng} / \mathrm{L}$ ) at the end of the followup period. The mean TSH level before high-dose T4 treatment was $1.8 \pm 0.4 \mathrm{mU} / \mathrm{L}$ and the final values were no longer measurable in all patients $(<.01 \mathrm{mU} / \mathrm{L})$. Even in the three patients who had already received physiological T4 supplementation before the T4 dose was raised the TSH levels were not suppressed.
Thus, almost all the thyroid hormone and TSH concentrations before initiation of high-dose T4 treatment were well within the normal range in all patients. However, the three patients who had not been given thyroxine before the start of the study (patients 4 through 6) had thyroid hormone concentrations at or even below the lower limit of the normal range in several of the numerous laboratory tests performed (full data not shown). These three patients repeatedly had T4 levels below $40 \mu \mathrm{g} / \mathrm{L}$ and T3 levels below $0.8 \mu \mathrm{g} / \mathrm{L}$ without any corresponding changes in TSH concentrations.

The side effects were negligible. Two patients complained of continuous mild sweating, and patient 4 had mild tachycardia ( 80 to 90 beats $/ \mathrm{min}$ ). The remaining four patients did not report any side effects that could have been related to the high-dose thyroxine treatment.

\section{DISCUSSION}

In our opinion, treatment of these very seriously ill and previously treatment-resistant patients with supraphysiological doses of thyroxine proved highly successful. After final adjustment of the doses of both T4 and the concomitant medication our patients had no further relapses. This leads us to conclude that-in addition to the treatment of refractory rapid-cycling bipolar patients-high-dose T4 supplementation may also be justified in severely treatment- and prophylaxisresistant, non-rapid cycling bipolar patients.

Both the experience gained in other previous studies (Stancer and Persad 1982; Bauer and Whybrow 1990) and our own results strongly suggest that supraphysiological levels of T4 should be given and supraphysiological serum concentrations of thyroid hormone attained in these patients. The fact that three of our patients ( 1 through 3 ) had already been receiving substitution therapy with physiological doses of T4 before we started them on supraphysiological doses and still suffered from treatment-resistant affective episodes strongly supports this conclusion.

As already suggested by Bauer and Whybrow (1990) we also believe that in the majority of cases high-dose T4 treatment alone - without concomitant psychotropic medication -is not effective. Patients 2, 3, 4, and 6 all received lithium and/or carbamazepine throughout the study period, but reducing the dose of antidepressants regularly induced symptoms of depression and reducing the dose of neuroleptics led to hypomanic symptoms. Therefore, at least in our patients, adding antidepressants (in cases in which the symptoms are mainly depressive) or neuroleptics (to prevent mania) seemed necessary. Whether or not lithium or carbamazepine treatment may also be important for maintaining the prophylactic effects of high-dose T4 was not inves- 
tigated in the present study. However, this has previously been suggested by Bauer and Whybrow (1990).

It is not yet clear why the interaction of pharmacotherapy with high-dose T4 is helpful in cases in which both forms of treatment given as monotherapy have failed. However, the following hypothesis is probably worth further investigation. In contrast to that in peripheral tissues, such as the liver and kidney, the supply of thyroid hormones to the central nervous system (CNS) does not depend on direct cellular uptake of T3 from the circulation but is almost entirely derived from intracellular deiodination of T4 (e.g., Crantz et al. 1982). Thus, the T4 supply to the CNS depends heavily on the serum levels of T4, and not on those of T3. Furthermore, we recently found that antidepressants such as desipramine (Baumgartner et al. 1990; Campos-Barros et al. 1994) and fluoxetine (Baumgartner et al. 1994), and also lithium and carbamazepine (Baumgartner et al., manuscript in preparation) enhance the activity of the iodothyronine $5^{\prime} \mathrm{II}$ deiodinase $\left(5^{\prime} \mathrm{D}\right.$-II $)$ isoenzyme in different regions of the CNS. This enzyme catalyzes the deiodination of the inactive prohormone $\mathrm{T} 4$ to the active metabolite T3 in the CNS (for a review, see Köhrle et al. 1991). As a result, all of these agents should lead to increases in tissue concentrations of $\mathrm{T} 3$, an effect that had already been observed after treatment with desipramine (Campos-Barros and Baumgartner 1994) and that may be related to its antidepressant properties. It would therefore seem reasonable to hypothesize that the different antidepressant/prophylactic agents must interact with the metabolism of T4 in the CNS before they can unfold their specific actions. In other words, both some antidepressants and lithium and carbamazepine may "need" thyroxine. On the other hand, all of these drugs reduce serum levels of thyroxine in depressed patients (see Roy-Byrne et al. 1984; Baumgartner et al. 1988; Kushner and Wartowsky 1988; Joffe and Singer 1990). In some of these studies the declines in serum concentrations of $\mathrm{T} 4$ were even correlated to the clinical efficacies of the respective drugs (e.g., Roy-Byrne et al. 1984; Baumgartner et al. 1988; Joffe and Singer 1990). These falls in serum levels probably result from increased T4 deiodination in tissues such as the CNS and the ensuing rise in tissue uptake of $\mathrm{T} 4$ from the circulation. A fall in the serum levels of T4 beyond a critical point might, on the other hand, interfere with the efficacy of these drugs, especially in patients whose serum levels of $\mathrm{T} 4$ are already at the lower limit of the normal range before treatment with psychotropic drugs is instituted. It is therefore interesting that all of the three patients who had not already been receiving T4 substitution before the institution of high-dose T4 treatment had serum levels at, or sometimes even below, the lower limit of the normal range.

Which biochemical mechanisms may underlie a possible antidepressant effect of $\mathrm{T} 3$ is unknown. How- ever, it seems worthy of note that there are numerous interactions between thyroid hormones and the characteristics of those transmitter receptors currently considered to be involved in the pharmacological action of antidepressant treatments, such as norepinephrine and serotonin. As early as 1981 Whybrow and Prange developed a hypothesis on the importance of interactions between catecholamines and thyroid hormones in the brain in affective disorders. They postulated that thyroid hormones act as neuromodulators, increasing beta-adrenergic function and reducing alpha-adrenergic activity. However, to date there remains some confusion as to what kind of receptor changes are induced by hypothyroidism and hyperthyroidism respectively, in which organs and in which species (for a discussion, see Baumgartner et al. 1988). Likewise, the effects of antidepressant treatments on noradrenergic and serotonergic receptor characteristics have proven to be far more complicated than was originally assumed, and thus it is not yet possible to draw any valid conclusions regarding a possible antidepressant effect of T3 mediated by these receptors.

It is striking that all four of the women in our study, but not the two men, had a history of subclinical hypothyroidism and/or Hashimoto's thyroiditis. In at least three of them, the thyroid disorder clearly preceded the lithium treatment. There are numerous reports in the literature of an abnormally high incidence of subclinical hypothyroidism in rapid-cycling bipolar patients, but the results are contradictory (e.g., Bauer et al. 1990; Wehr et al. 1988). Joffe et al. (1988) also investigated a group of non-rapid cycling bipolar patients and found that the incidence $(19 \%)$ of subclinical hypothyroidism in this subgroup was related to female sex and the duration of lithium treatment rather than to the diagnosis per se. In this regard it would seem interesting that Stancer and Persad (1982) reported that high-dose T4 treatment is effective only in rapid-cycling women, and not rapid-cycling men. In contrast, in our population, both men and women responded equally well.

Finally, it is interesting that our patients complained hardly at all of side effects, although their serum concentrations of thyroid hormone were in the supraphysiological range. It would therefore seem justified to ask whether these patients were suffering from a disturbance of some step between $\mathrm{T} 4$ secretion and T3 receptor effects.

\section{ACKNOWLEDGMENT}

The authors thank Ilse Becker, Martha Mau, Marianne ElSharif, and Régine von Schlippenbach from our outpatient clinics for their excellent cooperation. 


\section{REFERENCES}

Bauer MS, Whybrow PC (1990): Rapid cycling bipolar affective disorder. II. Treatment of refractory rapid cycling with high-dose levothyroxine: A preliminary study. Arch Gen Psychiatry 47:435-440

Bauer MS, Whybrow PC, Winokur A (1990): Rapid cycling bipolar affective disorder. I. Association with grade I hypothyroidism. Arch Gen Psychiatry 47:427-432

Baumgartner A, Gräf KJ, Kürten I, Meinhold H (1988): The hypothalamic pituitary thyroid axis in psychiatric patients and healthy subjects. Part II. Repeated measurements of thyroxine, free thyroxine, triiodothyronine, free triiodothyronine, and reverse triiodothyronine in patients with major depressive disorder and schizophrenia and healthy subjects. Psychiatry Res 24:283-305

Baumgartner A, Campos-Barros A, Stula M, Meinhold H (1990): Chronic desimipramine treatment enhances thyroxine deiodination in rat brain. Psychiatry Res 34:217219

Baumgartner A, Dubeyko M, Campos-Barros A, Eravci M, Meinhold H (1994): Subchronic administration of fluoxetine to rats affects triiodothyronine production and deiodination in regions of the cortex and in the limbic forebrain. Brain Res 635:68-74

Campos-Barros A, Baumgartner A (1994): Effects of chronic desipramine treatment on thyroid hormone concentrations in rat brain: Dependency on drug dose and brain area. Biol Psychiatry 35:214-216

Campos-Barros A, Meinhold H, Stula M, Müller F, Köhler R, Eravci M, Putzien O, Baumgartner A (1994): The influence of desipramine on thyroid hormone metabolism in rat brain. J Pharmacol Exp Ther (in press)

Crantz FR, Silva JE, Larsen PR (1982): Analysis of the sources and quantity of $3,5,3^{\prime}$-iodothyronine specifically bound to nuclear receptors in rat cerebral cortex and cerebellum. Endocrinology 110:367-375

Dunner D, Fieve R (1974): Clinical factors in lithium carbonate prophylaxis failure. Arch Gen Psychiatry 30:229-233

Feighner JP, King LJ, Schuckit MA, Croughan J, Briscoe W (1972): Hormonal potentiation of imipramine and ECT in primary depression. Amer J Psychiat 128:1230-1238

Gitlin MJ, Weiner H, Fairbanks L, Hershman JM, Friedfeld $\mathrm{N}$ (1987): Failure of T3 to potentiate tricyclic antidepressant response. J Affect Dis 13:267-272

Goodwin FK, Prange AJ, Post RM, Muscettola G, Lipton MA
(1982): Potentiation of antidepressant effects by L-triodothyronine in tricyclic nonresponders. Am J Psychiatry 139:34-38

Hall RC, Stickney S, Baerrisford TP (1986): Endocrine disease and behaviour. Integr Psychiatry 4:122-133

Joffe RT, Kutcher S, MacDonald C (1988): Thyroid function and bipolar affective disorder. Psychiatry Res 25:117-121

Joffe TR, Singer W (1990): Comparison of triiodothyronine and thyroxine in the potentiation of tricyclic antidepressants. Psychiatry Res 32:241-251

Köhrle J, Hesch D, Leonard JL (1991): Intracellular pathways of iodothyronine metabolism. In Bravemann LE, Utiger RD (eds), The Thyroid. Philadelphia, Lippincott, pp 144

Kushner JP, Wartofsky L (1988): Lithium-thyroid interactionsan overview. In Johnson FN (ed), Lithium and the Endocrine System, Vol. 2 Lithium Therapy Monographs. Basel/München/Paris, Karger, pp 74-98

Leibow D (1983): L-thyroxine for rapid-cycling bipolar illness. Amer J Psychiatry 140:1255

Prange AJ, Wilson IC, Rabon AM, Lipton MA (1969): Enhancement of imipramine anti-depressant activity by thyroid hormone. Amer J Psychiatry 126:457-469

Prange AJ, Wilson IC, Knox A, McClane TK, Lipton MA (1970): Enhancement of imipramine by thyroid stimulating hormone: Clinical and theoretical implications. Amer J Psychiatry 127:191-199

Prange AJ, Wilson IC, Lara PP, Alltop LB, Breese GR (1972): Effects of thyrotropin-releasing hormone in depression: Lancet ii:999-1002

Roy-Byrne PP, Joffe RT, Uhde TW, Post RM (1984): Carbamazepine and thyroid function in affectively ill patients. Arch Gen Psychiatry 41:1150-1153

Stancer HC, Persad E (1982): Treatment of intractable rapidcycling manic-depressive disorder with levothyroxine. Arch Gen Psychiatry 39:311-312

Stein D, Avni J (1988); Thyroid hormones in the treatment of affective disorders. Acta Psychiatr Scand 77:623-636

Wehr TA, Sack DA, Rosenthal NE, Cowdry RW (1988): Rapid cycling affective disorder: Contributing factors and treatment responses in 51 patients. Am J Psychiatry 145: 179-184

Whybrow PC, Prange AJ (1981): A hypothesis of thyroidcatecholamine-receptor interaction. Arch Gen Psychiatry 38:106-113 